\title{
Some robust design strategies for percentile estimation in binary response models
}

\author{
Stefanie Biedermann \\ Ruhr-Universität Bochum \\ Fakultät für Mathematik \\ 44780 Bochum \\ Germany
}

email: stefanie.biedermann@ruhr-uni-bochum.de

\author{
Holger Dette \\ Ruhr-Universität Bochum \\ Fakultät für Mathematik \\ 44780 Bochum \\ Germany
}

email: holger.dette@ruhr-uni-bochum.de

\author{
Andrey Pepelyshev \\ St. Petersburg State University \\ Department of Mathematics \\ St. Petersburg \\ Russia \\ email: andrey@ap7236.spb.edu
}

March 15, 2004

\begin{abstract}
For the problem of percentile estimation of a quantal response curve, we determine multi-objective designs which are robust with respect to misspecifications of the model assumptions. We propose a maximin approach based on efficiencies and provide designs that are simultaneously efficient with respect to the particular choice of various parameter regions and link functions. Furthermore, we deal with the problems of designing model and percentile robust experiments and give various examples of such designs, which are calculated numerically.
\end{abstract}

AMS Classification: 62K05, 62J12

Keywords and Phrases: Binary response model, robust optimal design, c-efficiency, percentile estimation, multi-objective designs. 


\section{Introduction}

We consider an experiment where the response is dichotomous and the probability of toxicity $p$ increases with the dose level $x$ of a drug. A $100 p$ th percentile $x_{p}$ is the dose level corresponding to a desired probability of toxicity $p$, i.e. for a sample space of doses $\mathcal{X} \subset \mathbb{R}$ the percentile $x_{p} \in \mathcal{X}$ corresponds to a target probability of toxicity $p \in(0,1)$. Estimation may be facilitated by assuming that the response function follows some probability distribution from a location scale family. If we let the probability of toxicity at dose level $x_{i}$ be modeled as $F\left(\beta x_{i}+\mu\right)$, i.e.

$$
p=p(x, \vartheta)=F(\mu+\beta x), \quad \vartheta=(\mu, \beta)^{T}, \mu \in \mathbb{R}, \beta \in \mathbb{R}^{+},
$$

where $\mu$ and $\beta$ are unknown parameters and $F$ is a distribution function, then we obtain the following expression for the $100 p$ th percentile

$$
x=x_{p}=\frac{F^{-1}(p)-\mu}{\beta} .
$$

An (approximate) design $\xi$ is a probability measure with finite support on $\mathbb{R}$, i.e. the observations are taken at the support points of the measure proportional to the corresponding masses. An optimal design maximizes a real-valued function of the Fisher information matrix, which is usually referred to as an optimality criterion [see e.g. Silvey (1980)]. In the present context, the Fisher information for the parameter $\vartheta$ depends on the unknown value of $\vartheta$ itself, which complicates the determination of optimal designs substantially. Much effort has been devoted to the problem of finding good designs for the estimation of the parameter $\vartheta$ in the binary response model [see, e.g., Chaloner and Larntz (1989), Sitter and Wu (1993) among many others].

The goal of this article is to provide designs $\xi$, which are on the one hand efficient for estimating the $100 p$ th percentile $x_{p}$ and on the other hand robust against misspecifications of the model assumptions. $\mathrm{Wu}(1988)$ shows that designing an experiment optimal for percentile estimation is equivalent to calculating the $c$-optimal design for the estimation of a linear combination of the parameters $\mu$ and $\beta$ with respect to a particular choice of the vector $c$. Locally (in the sense of Chernoff (1953)) c-optimal designs for the above model can be derived from the article of Ford, Torsney and Wu (1992). Since the Fisher information and thus the $c$-optimal designs depend on the unknown parameter these designs cannot be implemented directly in practice. Assuming that a good initial guess for the unknown parameter $\vartheta$ is available is often not realistic in real applications. Moreover, misspecifications of the parameter $\vartheta$ for the construction of a design can lead to a loss of efficiency [see, e.g., López-Fidalgo and Wong (2000)] and as a consequence to poor results in the subsequent data analysis. A more robust alternative is to assume sufficient knowledge of $\vartheta$ to specify a prior distribution for this parameter and to average the optimality criterion (or an appropriately standardized version thereof) over the plausible values of $\vartheta$ defined by the prior. This leads to so-called Bayesian optimality criteria [see e.g. Chaloner and Larntz (1989)].

As an alternative for the construction of robust designs, we propose a maximin approach based on $c$-efficiencies, which only requires the specification of a certain range for the unknown parameter. It appears to us that this is a more realistic scenario since practitioners will often 
have difficulties to specify a prior distribution for the unknown parameter $\vartheta$ especially if this is two-dimensional. A (non-standardized) maximin approach has been used by Sitter (1992) and Noubiap and Seidel (2000) for the problem of parameter estimation in binary response models. In contrast to these authors, we discuss a minimax concept based on efficiencies, because as pointed out in Dette (1997) the consideration of non-standardized quantities can be rather misleading.

In section 2, we describe the model in detail and derive the connection between optimality for percentile estimation and c-optimality. In section 3, the robust optimality criterion used in the following is illustrated and maximin efficient designs are given for several choices of spaces for the unknown parameter $\vartheta$. These results are then compared to the corresponding Bayesian optimal designs with respect to the uniform prior distribution. While the problem of robustness of an optimal design with respect to the unknown parameter is caused by the nonlinear structure of the binary response model, there are other issues of robustness which arise in applications, because a specific model or a particular percentile is chosen, for which the determined design should be efficient. For this reason, section 4 extends the maximin efficiency approach, dealing with maximin efficient designs for percentile estimation for competing link functions. In section 5, we present some results on designs, which are maximin efficient for estimating several percentiles simultaneously. A somewhat related approach is given in Zhu and Wong (2000), who derive designs, which are (non-standardized) Bayesian $c$-optimal (with respect to several prior distributions for the unknown parameter $\vartheta$ ) for the estimation of $x_{0.5}$, the LD50, under the constraint that the Bayesian efficiencies for estimating $x_{0.25}$ and $x_{0.75}$ separately will not be lower than some given values. The concluding section 6 , finally, summarizes our results and gives a detailed discussion of the various aspects of robustness in the design of experiment for the binary response setup.

\section{Locally optimal designs}

Before we present our results on maximin efficient designs for percentile estimation, we give a short summary of the results on the corresponding locally optimal designs, which were already established by $\mathrm{Wu}$ (1988). We consider the common binary response model where a subject is administered a stimulus at a dose level $x \in \mathbb{R}$. The response $X$ is a binary random variable with success probability $p=p(x, \vartheta)$, i.e. $X \sim \operatorname{Bin}(1, p(x, \vartheta))$, where $x \in \mathbb{R}$ is the explanatory variable and $\vartheta$ is an unknown parameter. In this article, we deal with parameterization (1.1) of a two parameter binary response model, where $F$ denotes a so-called link function, i.e. a known distribution function with density $f$. Having chosen a distribution family for $F$, one obtains maximum likelihood estimators $\hat{\mu}, \hat{\beta}$ for the unknown parameters and $\hat{x}_{p}=\left(F^{-1}(p)-\hat{\mu}\right) / \hat{\beta}$ for the $100 p$ th percentile. The information matrix for a single dose $x_{i}$ associated with $\hat{\mu}$ and $\hat{\beta}$ is given by

$$
I\left(x_{i}, \vartheta\right)=h\left(z_{i}\right)\left(\begin{array}{cc}
1 & x_{i} \\
x_{i} & x_{i}^{2}
\end{array}\right)
$$


where $z_{i}=\beta x_{i}+\mu$, and the function $h(z)$ is defined as

$$
h(z)=\frac{f^{2}(z)}{F(z)(1-F(z))} .
$$

The Fisher information of a design $\xi$ is thus given by

$$
M(\xi, \vartheta)=\int I(x, \vartheta) d \xi(x)
$$

and also called information matrix in the design literature. The notations $I(x, \vartheta), M(\xi, \vartheta)$ indicate the dependence of the Fisher information on the particular value of the unknown parameter $\vartheta$. By inverting the information matrix $M(\xi, \vartheta)$ of the underlying design $\xi$, we obtain a first-order approximation to the variance-covariance matrix of the maximum likelihood estimate $(\hat{\mu}, \hat{\beta})^{T}$. If $n$ observations are taken according to an approximate design (using an appropriate rounding procedure), we derive an approximation to the asymptotic variance of $\hat{x}_{p}$ as

$$
\operatorname{Var}\left(\hat{x}_{p}\right) \approx \frac{1}{n}\left(\nabla x_{p}\right)^{T} M^{-}(\xi, \vartheta)\left(\nabla x_{p}\right), \quad \nabla x_{p}=\left(\frac{\partial x_{p}}{\partial \mu}, \frac{\partial x_{p}}{\partial \beta}\right)^{T},
$$

where the gradient of $x_{p}$ is given by $c=c_{p}=-\left(1, x_{p}\right)^{T} / \beta$ and $M^{-}$denotes a generalized inverse of the matrix $M$. Here and throughout this paper, we assume that the linear combination $c_{p}^{T} \vartheta$ is estimable by the design, i.e. $c_{p} \in \operatorname{range}(M(\xi, \vartheta))$ for all $\vartheta \in \Theta$, which guarantees that the approximation for the variance of $\hat{x}_{p}$ is independent of the choice of the generalized inverse [see Pukelsheim (1993), chapter 3]. An optimal design for estimating the 100pth percentile minimizes $\operatorname{Var}\left(\hat{x}_{p}\right)$ or its approximation. Thus the problem of finding a (locally) optimal design for estimating $x_{p}$ is equivalent to the problem of finding a locally $c_{p}$-optimal design, i.e. a design which minimizes

$$
c_{p}^{T} M^{-}(\xi, \vartheta) c_{p}
$$

These designs are called locally optimal, since the minimization of (2.5) depends on the parameter $\vartheta=(\mu, \beta)^{T}$ [see Chernoff (1953)].

$\mathrm{Wu}(1988)$ shows that the $c_{p}$-optimal design problem for the binary response model (1.1) can be solved simultaneously for all parameters. There exist values $p_{1}<p_{2}$ depending on the link function $F$ such that for $p_{1} \leq p \leq p_{2}$ the locally $c_{p}$-optimal design is the one-point design concentrating at the $100 p$ th percentile $x_{p}$ defined in (1.2), whereas for $p<p_{1}$ or $p>p_{2}$ the locally $c_{p}$-optimal design is supported on the two percentiles $x_{p_{1}}$ and $x_{p_{2}}$, while the corresponding weights can be calculated from an explicit formula depending on $p, p_{1}, p_{2}$ and the particular choice of $F$. For the logit link function we obtain the values $p_{2}=1-p_{1}=0.917$. The probit link function yields $p_{2}=1-p_{1}=0.942$. Note that while the design points $x_{p_{1}}$ and $x_{p_{2}}$ depend on the unknown parameter $\vartheta$, the values $p_{1}$ and $p_{2}$ do only depend on the link function $F$ under consideration.

The most commonly applied link functions are the logistic distribution (logit) and the normal distribution (probit) and we will restrict ourselves to these two link functions in the following. Other links, which are also used in practice, are the complementary log-log, the double exponential, the double reciprocal and the skewed logit with several choices of skewing parameters [see Ford, Torsney and Wu (1992)] and can be treated by similar methods. 


\section{Efficient designs for percentile estimation under a given model assumption}

A general objection to one-point designs is that they do not allow the estimation of both parameters $\mu$ and $\beta$ with the same data. In the context of sequential experiments, however, such designs were shown to be robust to model assumptions [see Wu (1985)]. A disadvantage of collecting data sequentially is that they exhibit dependencies, which can lead to problems when it comes to data analysis.

Thus, we consider a non-sequential way of obtaining efficient designs for percentile estimation, which are robust against misspecifications of the unknown parameter $\vartheta=(\mu, \beta)^{T}$. Our approach is motivated by our experience that in many cases it is possible to specify a certain range, $\Theta \subset \mathbb{R} \times \mathbb{R}^{+}$say, for the unknown parameters. Following Müller (1995), we propose to use designs, which maximize the minimal $c_{p}$-efficiency, min eff $_{\Theta}(\xi)$, over the set $\Theta$, thus protecting the experiment against the worst case scenario. In other words, we propose to maximize the expression

$$
\min \operatorname{eff}_{\Theta}(\xi)=\min _{\vartheta \in \Theta} \operatorname{eff}_{c_{p}}(\xi, \vartheta)=\min _{\vartheta \in \Theta} \frac{c_{p}^{T} M^{-}\left(\xi_{c_{p}}^{*}, \vartheta\right) c_{p}}{c_{p}^{T} M^{-}(\xi, \vartheta) c_{p}}
$$

with respect to the choice of the design $\xi$, where $\xi_{c_{p}}^{*}$ denotes the locally $c_{p}$-optimal design and $M^{-}\left(\xi_{c_{p}}^{*}, \vartheta\right)$ is a generalized inverse of the information matrix $M\left(\xi_{c_{p}}^{*}, \vartheta\right)$. Since the locally $c_{p}$-optimal designs are one point designs in many cases, this definition takes singular Fisher information matrices into account. Note that the vector $c_{p}$ and the design $\xi_{c_{p}}^{*}$ depend on the parameter $\vartheta$, although this dependence is not reflected in our notations. Throughout this section a maximin efficient design will be denoted by $\xi_{M}^{*}$.

A powerful tool for checking the optimality of a given design are equivalence theorems. The equivalence theorem for local $c_{p}$-optimality, which is given below, is well-known in the design literature. The proof can, for example, be found in Pukelsheim (1993).

Theorem 3.1 A design $\xi_{c_{p}}^{*}=\xi_{c_{p}}^{*}(\vartheta)$ is locally $c_{p}$-optimal if and only if there exists a generalized inverse $G\left(\xi_{c_{p}}^{*}, \vartheta\right)$ of $M\left(\xi_{c_{p}}^{*}, \vartheta\right)$ such that the following inequality holds

$$
\frac{c_{p}^{T} G\left(\xi_{c_{p}}^{*}, \vartheta\right) I(x, \vartheta) G\left(\xi_{c_{p}}^{*}, \vartheta\right) c_{p}}{c_{p}^{T} M^{-}\left(\xi_{c_{p}}^{*}, \vartheta\right) c_{p}} \leq 1 \quad \forall x \in \mathbb{R}
$$

Moreover, there is equality in (3.2), when $x$ is a support point of the design $\xi_{c_{p}}^{*}$.

The equivalence theorem corresponding to the maximin $c_{p}$-efficiency criterion can be obtained as a special case of Theorem $3.3 \mathrm{~b}$ ) in Dette, Haines and Imhof (2003).

Theorem 3.2 Let

$$
\mathcal{N}(\xi)=\left\{\tilde{\vartheta} \in \Theta \mid \operatorname{eff}_{c_{p}}(\xi, \tilde{\vartheta})=\min _{\vartheta \in \Theta} \operatorname{eff}_{c_{p}}(\xi, \vartheta)\right\}
$$


be the subset of $\Theta$ consisting of those values of $\vartheta$, for which the $c_{p}$-efficiency of a design $\xi$ takes its minimal value over $\Theta$. A design $\xi_{M}^{*}$ is maximin $c_{p}$-efficient if and only if for each $\vartheta \in \mathcal{N}\left(\xi_{M}^{*}\right)$ there exists a generalized inverse $G\left(\xi_{M}^{*}, \vartheta\right)$ of $M\left(\xi_{M}^{*}, \vartheta\right)$ and a prior $\pi^{*}$ on $\mathcal{N}\left(\xi_{M}^{*}\right)$ such that the following inequality is valid

$$
\int_{\mathcal{N}\left(\xi_{M}^{*}\right)} \frac{c_{p}^{T} G\left(\xi_{M}^{*}, \vartheta\right) I(x, \vartheta) G\left(\xi_{M}^{*}, \vartheta\right) c_{p}}{c_{p}^{T} M^{-}\left(\xi_{M}^{*}, \vartheta\right) c_{p}} d \pi^{*}(\vartheta) \leq 1 \quad \forall x \in \mathbb{R} .
$$

Again, there is equality in (3.4), when $x$ is a support point of the design $\xi_{M}^{*}$.

Following Dette, Haines and Imhof (2003) we call any prior $\pi^{*}$ in (3.4) least favourable distribution. The designs given in the following discussion were calculated numerically using Theorem 3.2 whereby their optimality was carefully checked. These calculations are quite complicated, since the least favourable distribution is two-dimensional.

From a practical point of view, it is reasonable to assume that the experimenter can specify a certain range for the position of either parameter before the experiment. This information leads to a rectangular subset of $\mathbb{R} \times \mathbb{R}^{+}$for $\Theta$, i.e. $\Theta=\left[\mu_{1}, \mu_{2}\right] \times\left[\beta_{1}, \beta_{2}\right]$. In the following, we will thus consider only parameter regions $\Theta$ of this form. Lemma 3.1 and Lemma 3.2 below give considerable simplifications of the maximin optimal design problem for parameter regions of the form $\Theta=\left[\mu_{1}, \mu_{2}\right] \times\left[\beta_{1}, \beta_{2}\right]$. The first result describes a symmetry property of the maximin efficient designs for estimating the percentiles $x_{p}$ and $x_{1-p}$ in case of an underlying symmetric distribution function $F$.

Lemma 3.1 Let $F$ be a symmetric distribution function, i.e. $F(x)=1-F(-x)$. Let, furthermore, $\xi=\left\{x_{1}, \ldots, x_{n} ; w_{1}, \ldots, w_{n}\right\}$ denote the maximin efficient design for estimating the percentile $x_{p}$ with respect to the parameter space $\Theta=\left[\mu_{1}, \mu_{2}\right] \times\left[\beta_{1}, \beta_{2}\right]$. Then the design $\left\{-x_{1}, \ldots,-x_{n} ; w_{1}, \ldots, w_{n}\right\}$ is maximin efficient for estimating the percentile $x_{1-p}$ with respect to $\left[-\mu_{2},-\mu_{1}\right] \times\left[\beta_{1}, \beta_{2}\right]$.

Proof. Combining the equalities

$$
c_{p}=c_{p}(\mu, \beta)=-\left(1, x_{p}\right)^{T} / \beta=-\left(1, \frac{F^{-1}(p)-\mu}{\beta}\right)^{T} / \beta=\left(\begin{array}{cc}
1 & 0 \\
0 & -1
\end{array}\right) c_{1-p}(-\mu, \beta)
$$

and

$$
\begin{array}{r}
M(\xi, \mu, \beta)=\sum_{i} w_{i} \frac{f\left(\beta x_{i}+\mu\right)^{2}}{F\left(\beta x_{i}+\mu\right)\left(1-F\left(\beta x_{i}+\mu\right)\right)}\left(\begin{array}{cc}
1 & x_{i} \\
x_{i} & x_{i}^{2}
\end{array}\right) \\
=\left(\begin{array}{cc}
1 & 0 \\
0 & -1
\end{array}\right) M\left(\left\{-x_{i}, w_{i}\right\},-\mu, \beta\right)\left(\begin{array}{cc}
1 & 0 \\
0 & -1
\end{array}\right)
\end{array}
$$

the assertion of Lemma 3.1 is obvious.

Our next result refers to the relationship between the maximin efficient designs for estimating the percentile $x_{p}$ with respect to parameter regions $\Theta$ of a certain form, i.e. if the $\beta$-intervals are scaled by a positive parameter $\gamma$ the maximin efficient designs for the new parameter spaces 
can be obtained by rescaling the "old" designs. This result shows that maximin efficient designs are equivariant with respect to different scalings of the explanatory variable $x$. Moreover, it also enables us to restrict ourselves to consider only $\beta$-intervals with one fixed parameter value, e.g. $\beta_{1}=1$.

Lemma 3.2 Let $\xi=\left\{x_{1}, \ldots, x_{n} ; w_{1}, \ldots, w_{n}\right\}$ denote the maximin efficient design for estimating the percentile $x_{p}$ with respect to the parameter region $\Theta=\left[\mu_{1}, \mu_{2}\right] \times\left[\beta_{1}, \beta_{2}\right]$. Then the design $\xi_{\gamma}=\left\{x_{1} / \gamma, \ldots, x_{n} / \gamma ; w_{1}, \ldots, w_{n}\right\}$ is maximin efficient for estimating the percentile $x_{p}$ with respect to the parameter region $\left[\mu_{1}, \mu_{2}\right] \times\left[\gamma \beta_{1}, \gamma \beta_{2}\right]$ for all choices of $\gamma>0$.

Proof. Combining the equalities

$$
c_{p}(\mu, \beta)=-\left(1, x_{p}\right)^{T} / \beta=-\left(1, \frac{F^{-1}(p)-\mu}{\beta}\right)^{T} / \beta=\gamma\left(\begin{array}{ll}
1 & 0 \\
0 & \gamma
\end{array}\right) c_{p}(\mu, \gamma \beta)
$$

and

$$
\begin{aligned}
M(\xi, \mu, \beta) & =\sum_{i} \frac{f\left(\beta x_{i}+\mu\right)^{2}}{F\left(\beta x_{i}+\mu\right)\left(1-F\left(\beta x_{i}+\mu\right)\right)}\left(\begin{array}{cc}
1 & x_{i} \\
x_{i} & x_{i}^{2}
\end{array}\right) \\
& =\left(\begin{array}{ll}
1 & 0 \\
0 & \gamma
\end{array}\right) M\left(\xi_{\gamma}, \mu, \gamma \beta\right)\left(\begin{array}{cc}
1 & 0 \\
0 & \gamma
\end{array}\right)
\end{aligned}
$$

we obtain the statement of Lemma 3.2.

It is obvious that maximin efficient designs must be supported on more than one point, since a one-point design does not allow the estimation of the percentile $x_{p}$ with respect to different unknown parameters. From a heuristic point of view it is also clear that if the length $\mu_{2}-\mu_{1}$ and $\beta_{2}-\beta_{1}$ of the parameter intervals is small then the maximin efficient designs for estimating percentiles $x_{p}$ with $p_{1} \leq p \leq p_{2}$ take the form $\left\{x_{1}, x_{2} ; w, 1-w\right\}$, where $x_{1} \approx x_{p}$ and $w \approx 1$ [see $\mathrm{Wu}(1988)]$. In other words, the maximin efficient design $\xi_{M}^{*}$ is close to the locally $c_{p}$-optimal designs with respect to $(\mu, \beta)^{T} \in\left[\mu_{1}, \mu_{2}\right] \times\left[\beta_{1}, \beta_{2}\right]$.

These presumptions are confirmed by our numerical results, which are given in Tables 1 and 3. Table 1 presents some maximin efficient designs with respect to several relatively small parameter intervals for the logit distribution

$$
F(x)=1 /\left(1+e^{-x}\right), \mathcal{X}=\mathbb{R},
$$

whereas Table 3 gives the corresponding designs for the probit distribution

$$
F(x)=\int_{-\infty}^{x} e^{-u^{2} / 2} d u / \sqrt{2 \pi}, \mathcal{X}=\mathbb{R} .
$$

For "large" parameter regions $\Theta$ we obtain maximin efficient designs with more than two support points (see Tables 2 and 4). This phenomenon is widely observed in the literature 
as far as Bayesian or maximin optimal design problems are concerned [see, e.g., Dette and Biedermann (2003) or Dette and Wong (1996)]. The expression

$$
b\left(\xi_{M}^{*}\right)=\int_{\Theta} \operatorname{eff}_{c_{p}}\left(\xi_{M}^{*}, \vartheta\right) d \vartheta / \lambda(\Theta)
$$

in the tables denotes the value of the integrated (with respect to the uniform distribution) $c_{p}$-efficiency of the maximin efficient design over the parameter region $\Theta$ and is given for comparison with Bayesian designs with respect to the uniform prior, which will be discussed below. [Here $\lambda(\Theta)$ denotes the Lebesgue measure of $\Theta$, i.e. $\lambda(\Theta)=\left(\mu_{2}-\mu_{1}\right)\left(\beta_{2}-\beta_{1}\right)$.]

Table 1: Maximin efficient designs for percentile estimation in the logit model with respect to various "small" parameter regions $\Theta=\left[\mu_{1}, \mu_{2}\right] \times\left[\beta_{1}, \beta_{2}\right]$. In these cases the optimal designs are supported at two points.

\begin{tabular}{|c|c|c|c|c|c|c|c|c|c|c|}
\hline$p$ & $\mu_{1}$ & $\mu_{2}$ & $\beta_{1}$ & $\beta_{2}$ & $x_{1}$ & $x_{2}$ & $w_{1}$ & $w_{2}$ & $\min \operatorname{eff}_{\Theta}\left(\xi_{M}^{*}\right)$ & $b\left(\xi_{M}^{*}\right)$ \\
\hline 0.2 & -0.1 & 0.1 & 1 & 1.1 & -1.381 & 0.903 & 0.938 & 0.062 & 0.8888 & 0.9342 \\
0.4 & -0.1 & 0.1 & 1 & 1.1 & -0.751 & 0.189 & 0.621 & 0.379 & 0.8986 & 0.9329 \\
0.5 & -0.1 & 0.1 & 1 & 1.1 & -0.423 & 0.423 & 0.500 & 0.500 & 0.9121 & 0.9385 \\
0.6 & -0.1 & 0.1 & 1 & 1.1 & -0.189 & 0.751 & 0.379 & 0.621 & 0.8986 & 0.9329 \\
0.7 & -0.1 & 0.1 & 1 & 1.1 & -0.363 & 0.992 & 0.149 & 0.851 & 0.8891 & 0.9303 \\
0.8 & -0.1 & 0.1 & 1 & 1.1 & -0.903 & 1.381 & 0.062 & 0.938 & 0.8888 & 0.9342 \\
\hline 0.9 & -0.1 & 0.1 & 1 & 1.1 & -1.914 & 1.964 & 0.054 & 0.946 & 0.9230 & 0.9609 \\
0.9 & -0.2 & 0.2 & 1 & 1.2 & -1.664 & 1.831 & 0.094 & 0.906 & 0.8359 & 0.9183 \\
0.9 & -0.3 & 0.3 & 1 & 1.3 & -1.375 & 1.756 & 0.124 & 0.877 & 0.7509 & 0.8769 \\
\hline 0.95 & -0.1 & 0.1 & 1 & 1.1 & -2.254 & 2.232 & 0.111 & 0.890 & 0.9728 & 0.9949 \\
0.95 & -0.2 & 0.2 & 1 & 1.2 & -2.104 & 2.061 & 0.141 & 0.859 & 0.9108 & 0.9758 \\
0.95 & -0.3 & 0.3 & 1 & 1.3 & -1.963 & 1.923 & 0.171 & 0.829 & 0.8349 & 0.9494 \\
\hline
\end{tabular}

Table 2: Maximin efficient designs for percentile estimation in the logit model with respect to various "moderate" and "large" parameter regions $\Theta=\left[\mu_{1}, \mu_{2}\right] \times\left[\beta_{1}, \beta_{2}\right]$.

\begin{tabular}{|c|c|c|c|c|c|c|c|c|c|c|c|c|c|c|}
\hline$p$ & $\mu_{1}$ & $\mu_{2}$ & $\beta_{1}$ & $\beta_{2}$ & $x_{1}$ & $x_{2}$ & $x_{3}$ & $x_{4}$ & $w_{1}$ & $w_{2}$ & $w_{3}$ & $w_{4}$ & $\min _{\mathrm{eff}}\left(\xi_{M}^{*}\right)$ & $b\left(\xi_{M}^{*}\right)$ \\
\hline 0.5 & -1 & 1 & 1 & 2 & -1.812 & -0.675 & 0.675 & 1.812 & 0.144 & 0.356 & 0.356 & 0.144 & 0.4948 & 0.5941 \\
0.5 & -2 & 2 & 1 & 4 & -2.264 & -0.518 & 0.518 & 2.264 & 0.181 & 0.319 & 0.319 & 0.181 & 0.2519 & 0.3919 \\
\hline 0.8 & -1 & 1 & 1 & 2 & -0.126 & 0.986 & 2.333 & & 0.286 & 0.294 & 0.420 & & 0.4736 & 0.5898 \\
0.8 & -2 & 2 & 1 & 4 & -1.105 & -0.100 & 0.858 & 2.741 & 0.075 & 0.255 & 0.365 & 0.305 & 0.2609 & 0.4045 \\
\hline 0.9 & -0.4 & 0.4 & 1 & 1.4 & -1.666 & 0.184 & 1.876 & & 0.082 & 0.122 & 0.796 & & 0.6787 & 0.8136 \\
0.9 & -1 & 1 & 1 & 2 & 0.118 & 1.051 & 2.591 & & 0.197 & 0.307 & 0.496 & & 0.4756 & 0.5826 \\
0.9 & -2 & 2 & 1 & 4 & -0.121 & 0.420 & 1.200 & 3.111 & 0.164 & 0.221 & 0.307 & 0.308 & 0.3101 & 0.4133 \\
\hline
\end{tabular}


Table 3: Maximin efficient designs for percentile estimation in the probit model with respect to various "small" parameter regions $\Theta=\left[\mu_{1}, \mu_{2}\right] \times\left[\beta_{1}, \beta_{2}\right]$.

\begin{tabular}{|c|c|c|c|c|c|c|c|c|c|c|}
\hline$p$ & $\mu_{1}$ & $\mu_{2}$ & $\beta_{1}$ & $\beta_{2}$ & $x_{1}$ & $x_{2}$ & $w_{1}$ & $w_{2}$ & $\min \mathrm{eff}_{\Theta}\left(\xi_{M}^{*}\right)$ & $b\left(\xi_{M}^{*}\right)$ \\
\hline 0.2 & -0.1 & 0.1 & 1 & 1.1 & -0.891 & 0.615 & 0.906 & 0.094 & 0.8663 & 0.9185 \\
0.4 & -0.1 & 0.1 & 1 & 1.1 & -0.558 & 0.261 & 0.616 & 0.384 & 0.8848 & 0.9224 \\
0.5 & -0.1 & 0.1 & 1 & 1.1 & -0.382 & 0.382 & 0.500 & 0.500 & 0.8960 & 0.9269 \\
0.6 & -0.1 & 0.1 & 1 & 1.1 & -0.260 & 0.558 & 0.384 & 0.616 & 0.8848 & 0.9225 \\
0.7 & -0.1 & 0.1 & 1 & 1.1 & -0.371 & 0.682 & 0.189 & 0.811 & 0.8741 & 0.9193 \\
0.8 & -0.1 & 0.1 & 1 & 1.1 & -0.615 & 0.891 & 0.094 & 0.906 & 0.8663 & 0.9185 \\
\hline 0.9 & -0.1 & 0.1 & 1 & 1.1 & -1.066 & 1.202 & 0.059 & 0.941 & 0.8744 & 0.9305 \\
0.9 & -0.2 & 0.2 & 1 & 1.2 & -0.878 & 1.162 & 0.108 & 0.892 & 0.7541 & 0.8665 \\
0.9 & -0.3 & 0.3 & 1 & 1.3 & -0.680 & 1.148 & 0.150 & 0.850 & 0.6447 & 0.8087 \\
\hline 0.95 & -0.1 & 0.1 & 1 & 1.1 & -1.371 & 1.399 & 0.076 & 0.924 & 0.9243 & 0.9718 \\
0.95 & -0.2 & 0.2 & 1 & 1.2 & -1.216 & 1.290 & 0.123 & 0.877 & 0.8076 & 0.9221 \\
0.95 & -0.3 & 0.3 & 1 & 1.3 & -1.090 & 1.213 & 0.165 & 0.835 & 0.6893 & 0.8670 \\
\hline
\end{tabular}

We observe from Tables 1, 2, 3 and 4 that the support points of the optimal designs are much more spread on the real axis in case of an underlying logistic distribution than they are for the probit distribution. For several parameter regions $\Theta$ and percentiles $x_{p}$, the maximin $c_{p}$-efficient designs with respect to the probit model even require more support points than the respective designs for the logit link function (see Tables 2 and 4). Furthermore, the minimal efficiencies are higher in the logit case, particularly for larger parameter regions $\Theta$. These phenomena are probably due to differences in tail-behaviour of the link functions. We also observe that the maximin efficient designs yield very good minimal efficiencies if the space $\Theta=\left[\mu_{1}, \mu_{2}\right] \times\left[\beta_{1}, \beta_{2}\right]$ is not too large. For example, if the $95 \%$ percentile has to be estimated in the logit model, the minimal efficiency over the range $[-0.3,0.3] \times[1,1.3]$ of the maximin efficient design is $83.49 \%$. On the other hand, a larger uncertainty with respect to the location of the unknown parameters $(\mu, \beta)^{T}$ yields (as expected) a loss in minimal efficiency. Consider for example the problem of

Table 4: Maximin efficient designs for percentile estimation in the probit model with respect to various "moderate" and "large" parameter regions $\Theta=\left[\mu_{1}, \mu_{2}\right] \times\left[\beta_{1}, \beta_{2}\right]$.

\begin{tabular}{|c|c|c|c|c|c|c|c|c|c|c|c|c|c|c|}
\hline$p$ & $\mu_{1}$ & $\mu_{2}$ & $\beta_{1}$ & $\beta_{2}$ & $x_{1}$ & $x_{2}$ & $x_{3}$ & $x_{4}$ & $w_{1}$ & $w_{2}$ & $w_{3}$ & $w_{4}$ & $\min \mathrm{eff}_{\Theta}\left(\xi_{M}^{*}\right)$ & $b\left(\xi_{M}^{*}\right)$ \\
\hline 0.5 & -1 & 1 & 1 & 2 & -1.804 & -0.613 & 0.613 & 1.804 & 0.125 & 0.375 & 0.375 & 0.125 & 0.4272 & 0.5262 \\
0.5 & -2 & 2 & 1 & 4 & -1.507 & -0.452 & 0.452 & 1.507 & 0.260 & 0.240 & 0.240 & 0.260 & 0.1118 & 0.3007 \\
\hline 0.8 & -1 & 1 & 1 & 2 & -0.684 & 0.027 & 1.031 & 2.273 & 0.065 & 0.349 & 0.357 & 0.229 & 0.3877 & 0.4804 \\
0.8 & -2 & 2 & 1 & 4 & -0.994 & 0.001 & 0.712 & 1.946 & 0.174 & 0.291 & 0.275 & 0.260 & 0.1211 & 0.3045 \\
\hline 0.9 & -0.4 & 0.4 & 1 & 1.4 & -0.974 & 0.504 & 1.665 & & 0.024 & 0.440 & 0.537 & & 0.5611 & 0.6560 \\
0.9 & -1 & 1 & 1 & 2 & -0.381 & 0.161 & 1.022 & 2.165 & 0.041 & 0.306 & 0.359 & 0.294 & 0.3674 & 0.4537 \\
0.9 & -2 & 2 & 1 & 4 & -0.644 & 0.065 & 0.743 & 1.973 & 0.158 & 0.258 & 0.275 & 0.309 & 0.1283 & 0.2967 \\
\hline
\end{tabular}


estimating the median in a logit model where $\Theta=[-2,2] \times[1,4]$ is used for the construction of the maximin efficient design. In this case, the maximin efficient design is supported at four points and exhibits a minimal efficiency of $25.19 \%$. The reason for this low efficiency is that the maximin optimal design is a compromise between the rather extreme situations appearing in $\Theta$. For example if $\vartheta=(-2,1)^{T}$ or $\vartheta=(2,1)^{T}$, the locally optimal design advises the experimenter to take only observations at the point $x=2$ or $x=-2$, respectively, and the corresponding efficiencies for the other situation are both obtained as 19.63\%. Note that this value is already smaller than the minimal efficiency of the maximin optimal design, although only the value of $\mu$ was misspecified, whereas the efficiency of the maximin optimal design over the whole parameter region $\Theta$ is at least $25.19 \%$. Moreover, this value corresponds to the worst case with respect to $\Theta$ and for many parameter values of $\Theta$ the efficiency of the maximin optimal design is considerably higher. Imagine that the true value of $\vartheta$ is given by $\vartheta=(0,2)^{T}$ or $\vartheta=(0,1)^{T}$. Then the maximin optimal design achieves efficiencies $50.87 \%$ and even $72.05 \%$, respectively.

In the remaining part of this section, we want to address the application of Bayesian designs when the experimenter is in the situation that he can only specify a parameter region $\Theta$, in which the unknown parameter $\vartheta$ will be located, but he has not enough knowledge in advance of the experiment to assign plausible weights to the elements of $\Theta$. In this case, it is reasonable to give each point in $\Theta$ the same weight, i.e. to choose the uniform prior $d \vartheta / \lambda(\Theta)$ on the parameter region, where $\lambda(\Theta)$ denotes the Lebesgue measure of $\Theta$. The Bayesian $c_{p}$-efficient design $\xi_{B}^{*}$ with respect to the uniform prior $d \vartheta / \lambda(\Theta)$ thus maximizes the integral of the $c_{p}$-efficiencies with respect to $d \vartheta / \lambda(\Theta)$, i.e.

$$
\xi_{B}^{*}=\arg \max _{\xi} \int_{\Theta} \operatorname{eff}_{c_{p}}(\xi, \vartheta) d \vartheta / \lambda(\Theta)
$$

For a general Bayesian criterion the uniform prior $d \vartheta / \lambda(\Theta)$ has to be replaced by an arbitrary prior $\pi_{\Theta}$ on $\Theta$. The equivalence theorem corresponding to the above problem is given as a special case of Theorem 3.3 a) in Dette, Haines and Imhof (2003) and formulated for a general prior on $\Theta$.

Theorem 3.3 A design $\xi_{B}^{*}$ is Bayesian $c_{p}$-efficient with respect to a prior $\pi_{\Theta}$ if and only if for each $\vartheta \in \Theta$ there exists a generalized inverse $G\left(\xi_{B}^{*}, \vartheta\right)$ of $M\left(\xi_{B}^{*}, \vartheta\right)$ such that the following inequality holds for all $x \in \mathbb{R}$

$$
\int_{\Theta} \frac{c_{p}^{T} G\left(\xi_{B}^{*}, \vartheta\right) I(x, \vartheta) G\left(\xi_{B}^{*}, \vartheta\right) c_{p}}{c_{p}^{T} M^{-}\left(\xi_{B}^{*}, \vartheta\right) c_{p}} \operatorname{eff}_{c_{p}}\left(\xi_{B}^{*}, \vartheta\right) d \pi_{\Theta}(\vartheta) \leq \int_{\Theta} \operatorname{eff}_{c_{p}}\left(\xi_{B}^{*}, \vartheta\right) d \pi_{\Theta}(\vartheta)
$$

Moreover, there is equality in (3.6) for each support point of the Bayesian $c_{p}$-efficient design $\xi_{B}^{*}$.

The situation described above is exactly that way, for which we recommend to use the maximin $c_{p}$-efficiency criterion for the design of the experiment and it is of some interest to compare the designs with respect to the different concepts. In the following, we give some optimal designs with respect to the Bayesian $c_{p}$-efficency criterion with uniform prior distribution on $\Theta$ 
Table 5: Bayesian $c_{p}$-efficient designs for percentile estimation in the logit model with respect to the uniform prior distributions on various parameter regions $\Theta$.

\begin{tabular}{|c|c|c|c|c|c|c|c|c|c|c|}
\hline$p$ & $\mu_{1}$ & $\mu_{2}$ & $\beta_{1}$ & $\beta_{2}$ & $x_{1}$ & $x_{2}$ & $w_{1}$ & $w_{2}$ & $b\left(\xi_{B}^{*}\right)$ & $\min \operatorname{eff}_{\Theta}\left(\xi_{B}^{*}\right)$ \\
\hline 0.9 & -0.1 & 0.1 & 1 & 1.1 & -2.010 & 2.025 & 0.025 & 0.975 & 0.9715 & 0.8947 \\
0.9 & -0.2 & 0.2 & 1 & 1.2 & -1.845 & 1.898 & 0.043 & 0.957 & 0.9406 & 0.7765 \\
0.9 & -0.3 & 0.3 & 1 & 1.3 & -1.688 & 1.798 & 0.058 & 0.942 & 0.9093 & 0.6622 \\
0.9 & -0.4 & 0.4 & 1 & 1.4 & -1.535 & 1.717 & 0.070 & 0.930 & 0.8788 & 0.5583 \\
0.9 & -1 & 1 & 1 & 2 & -0.655 & 1.457 & 0.113 & 0.887 & 0.7283 & 0.1838 \\
\hline
\end{tabular}

Table 6: Bayesian $c_{p}$-efficient designs for percentile estimation in the probit model with respect to the uniform prior distributions on various parameter regions $\Theta$.

\begin{tabular}{|c|c|c|c|c|c|c|c|c|c|c|}
\hline$p_{1}$ & $\mu_{1}$ & $\mu_{2}$ & $\beta_{1}$ & $\beta_{2}$ & $x_{1}$ & $x_{2}$ & $w_{1}$ & $w_{2}$ & $b\left(\xi_{B}^{*}\right)$ & $\min \operatorname{eff}_{\Theta}\left(\xi_{B}^{*}\right)$ \\
\hline 0.9 & -0.1 & 0.1 & 1 & 1.1 & -1.134 & 1.210 & 0.026 & 0.974 & 0.9483 & 0.8287 \\
0.9 & -0.2 & 0.2 & 1 & 1.2 & -1.003 & 1.157 & 0.047 & 0.953 & 0.8988 & 0.6707 \\
0.9 & -0.3 & 0.3 & 1 & 1.3 & -0.884 & 1.116 & 0.066 & 0.934 & 0.8524 & 0.5354 \\
0.9 & -0.4 & 0.4 & 1 & 1.4 & -0.775 & 1.082 & 0.082 & 0.918 & 0.8093 & 0.4238 \\
0.9 & -1 & 1 & 1 & 2 & -0.298 & 0.945 & 0.172 & 0.829 & 0.6097 & 0.1037 \\
\hline
\end{tabular}

and compare these with the maximin designs given in Tables 1, 2, 3 and 4. Table 5 displays several Bayesian $c_{p}$-efficient designs for the logit model with respect to uniform priors on various parameter regions $\Theta$, where the expression $b\left(\xi_{B}^{*}\right)$ denotes the optimal criterion value, i.e.

$$
b^{*}\left(\xi_{B}^{*}\right)=\int_{\Theta} \operatorname{eff}_{c_{p}}\left(\xi_{B}^{*}, \vartheta\right) d \vartheta / \lambda(\Theta)
$$

and

$$
\min \operatorname{eff}_{\Theta}\left(\xi_{B}^{*}\right)=\min _{\vartheta \in \Theta} \operatorname{eff}_{c_{p}}\left(\xi_{B}^{*}, \vartheta\right)
$$

is the minimal $c_{p}$-efficiency of $\xi_{B}^{*}$ in $\Theta$. For the sake of brevity, we restrict ourselves to the problem of estimating the $90 \%$ percentile. The corresponding designs with respect to the probit model are given in Table 6.

Comparing the Bayesian $c_{p}$-efficient designs from Tables 5 and 6 with their maximin counterparts, we first notice that the Bayesian optimal designs have always two support points even for relatively large parameter regions $\Theta$. Moreover, one support point is assigned a very small weight so that the Bayesian optimal designs are close to the locally $c_{p}$-optimal designs on the corresponding parameter region $\Theta$, which are supported on one point only. This phenomenon is also observed for the maximin efficient designs, if the specified parameter space $\Theta$ is "small" (see Tables 1 and 3), but not for "larger" parameter spaces (see Tables 2 and 4). As expected from the definitions of the respective optimality criteria, the averaged efficiencies $b^{*}$ of 
the Bayesian optimal designs are somewhat higher than the averaged efficiencies of the maximin $c_{p}$-efficient designs. Turning to the comparison of minimal $c_{p}$-efficiencies, things change dramatically, especially for large parameter regions.

We consider, as a representative example, the optimal designs in the logit model for estimating the percentile $x_{p}$ with $p=0.9$ and assume that the experimenter is able to specify the parameter region $\Theta=[-1,1] \times[1,2]$. The averaged efficiency of the Bayesian optimal design is given by the value $b\left(\xi_{B}^{*}\right)=0.7283$, indicating high $c_{p}$-efficiencies for this design at most points in the parameter region $\Theta$, whereas the integrated efficiency of the corresponding maximin optimal design is given by $b\left(\xi_{M}^{*}\right)=0.5826$, which is of course smaller than the result for the Bayesian design, but still in acceptable limits. The result for the minimal $c_{p}$-efficiency of the Bayesian design, min $\operatorname{eff}_{\Theta}\left(\xi_{B}^{*}\right)=0.1838$, however, reveals that there are regions in $\Theta$, in which the Bayesian optimal design does not facilitate efficient estimation of the 100pth percentile $x_{p}$, i.e. the Bayesian design is not robust against every value $\vartheta \in \Theta$. The corresponding maximin efficient design, in contrast, achieves a minimal efficiency of 0.4756 , which is significantly higher than 0.1838. In this example, the Bayesian optimal design is supported on two points with much weight assigned to 1.457 , i.e. it is close to the locally optimal designs for parameters satisfying the equation $1.457 \beta=F^{-1}(0.9)-\mu$. For such parameters, we thus expect the design to perform highly efficient, whereas for parameter combinations from $\Theta$, which are located far away from the straight line described above, the efficiency of the Bayesian optimal design will be considerably smaller. Consider for example the parameter combinations $\mu=0, \beta=1.5$ and $\mu=-1, \beta=1$ from the parameter space $\Theta$, respectively. For the first parameter combination, which is located almost on the straight line, the Bayesian optimal design has efficiency 0.972, whereas its efficiency with respect to the second parameter combination turns out to be the unsatisfactory value of 0.189 . The maximin optimal design, which is much more spread on the real axis and thus not close to any locally optimal design at all, yields efficiencies 0.648 and 0.489 for the above parameters, respectively. Summarizing this result, we notice that for many values $\vartheta$ in the parameter region $\Theta$ the Bayesian optimal design will be slightly more efficient than the corresponding maximin optimal design, but there are elements $\vartheta \in \Theta$, for which efficient percentile estimation is almost impossible when using the Bayesian optimal design. It is also worthwhile to mention that maximin efficient designs often have more support points than the corresponding Bayesian optimal designs with respect to the uniform distribution on $\Theta$. This is an important advantage because additional support points facilitate to check the model assumption by goodness-of-fit tests (e.g. testing against a binary response model with three parameters).

For small parameter regions, the differences between Bayesian and maximin $c_{p}$-efficient designs are relatively small, so that it will make not much difference, for which criterion the experimenter decides. The larger the assumed parameter region (i.e. the higher the experimenter's uncertainty about the position of the parameter $\vartheta$ ), the stronger the use of the maximin $c_{p^{-}}$ efficiency criterion is recommended. 


\section{Efficient designs for percentile estimation in case of model uncertainty}

Besides the robustness problems of experimental designs with respect to the misspecification of some unknown non-linear model parameters, there are other issues of robustness, which have to be taken into account for the design of experiment. In particular the design also depends on the link function in (1.1), but in general it will be difficult for the experimenter to specify one distribution $F$ as the appropriate link function in advance. For example, it might not be obvious if the normal distribution or a more heavy-tailed link function such as the logit link would fit the data adequately before even collecting them. Misspecification of the quantal response model, however, may result in a large bias when estimating the percentiles of interest. It is thus sensible to choose the experimental conditions as in some sense "good" for several choices of links. Following Läuter (1974), we assume that the experimenter can define preferences $\alpha_{j}$ with respect to certain link functions $F_{j}$, where the weights $\alpha_{j}$ add up to one. Define $M_{(j)}(\xi, \vartheta)$ as the information matrix with respect to the quantal response model with link function $F_{j}$ and $\xi_{c_{p}(j)}^{*}$ as the corresponding locally $c_{p}(j)$-optimal design. Then an appropriate optimality criterion is of the form

$$
\sum_{j} \alpha_{j} \min _{\vartheta \in \Theta} \frac{c_{p}(j)^{T} M_{(j)}^{-}\left(\xi_{c_{p}(j)}^{*}, \vartheta\right) c_{p}(j)}{c_{p}(j)^{T} M_{(j)}^{-}(\xi, \vartheta) c_{p}(j)} \rightarrow \max _{\xi}
$$

The equivalence theorem, by which the optimality of a design with respect to criterion (4.1) can be checked, is given below and can be derived by combining parts a) and b) of Theorem 3.3 in Dette, Haines and Imhof (2003).

Theorem 4.1 A design $\xi^{*}$ is optimal with respect to the criterion defined in (4.1) if and only if there exist prior distributions $\pi_{j}^{*}$ on $\mathcal{N}_{j}\left(\xi^{*}\right)$ and for each $\vartheta \in \mathcal{N}_{j}\left(\xi^{*}\right)$ generalized inverses $G_{(j)}\left(\xi^{*}, \vartheta\right)$ of $M_{(j)}\left(\xi^{*}, \vartheta\right)$ such that the inequality

$$
\sum_{j} \alpha_{j} \int_{\mathcal{N}_{j}\left(\xi^{*}\right)} \frac{c_{p}(j)^{T} G_{(j)}\left(\xi^{*}, \vartheta\right) I(x, \vartheta) G_{(j)}\left(\xi^{*} \vartheta\right) c_{p}(j)}{c_{p}(j)^{T} M_{(j)}^{-}\left(\xi^{*}, \vartheta\right) c_{p}(j)} \operatorname{eff}_{c_{p}(j)}\left(\xi^{*}, \vartheta\right) d \pi_{j}^{*}(\vartheta) \leq u^{*}
$$

holds for all $x \in \mathbb{R}$. The expression eff $_{c_{p}(j)}(\xi, \vartheta)$ denotes the $c_{p}$-efficiency of the design $\xi$ with respect to the binary response model with link function $F_{j}, \mathcal{N}_{j}\left(\xi^{*}\right)$ is given by the set

$$
\mathcal{N}_{j}\left(\xi^{*}\right)=\left\{\tilde{\vartheta} \in \Theta \mid \operatorname{eff}_{c_{p}(j)}\left(\xi^{*}, \tilde{\vartheta}\right)=u_{j}^{*}\right\}
$$

and the values $u^{*}$ and $u_{j}^{*}$ are defined as

$$
u^{*}=\sum_{j} \alpha_{j} u_{j}^{*}, \quad u_{j}^{*}=\min _{\vartheta \in \Theta} \operatorname{eff}_{c_{p}(j)}\left(\xi^{*}, \vartheta\right)
$$

Moreover, there is equality in (4.2) for all support points of the design $\xi^{*}$. 
We consider the situation, in which both the probit and the logit link function are assigned weight $\alpha_{j}=1 / 2, j=1,2$, i.e. the experimenter has no preference with respect to one of these models, thus designing the experiment "equally well" for both choices of link functions. If the experimenter considers the logit link function more likely, he could e.g. assign weight 3/4 to this model and only $1 / 4$ to the probit model. Some results for different values of $p$ with respect to several parameter regions $\Theta$ are given in Table 7. Again, the optimality of these designs with respect to the criterion (4.1) has been verified by Theorem 4.1 .

Table 7: Maximin efficient designs for percentile estimation with respect to the optimality criterion (4.1) if the probit and the logit model are assigned weights $\alpha_{1}=\alpha_{2}=1 / 2$.

\begin{tabular}{|c|c|c|c|c|c|c|c|c|c|c|c|}
\hline$p$ & $\mu_{1}$ & $\mu_{2}$ & $\beta_{1}$ & $\beta_{2}$ & $x_{1}$ & $x_{2}$ & $w_{1}$ & $w_{2}$ & $u^{*}$ & $u_{1}^{*}$ & $u_{2}^{*}$ \\
\hline 0.5 & -0.1 & 0.1 & 1 & 1.1 & -0.398 & 0.398 & 0.5 & 0.5 & 0.9027 & 0.9105 & 0.8948 \\
0.6 & -0.1 & 0.1 & 1 & 1.1 & -0.025 & 1.163 & 0.645 & 0.355 & 0.8343 & 0.8591 & 0.8096 \\
0.7 & -0.1 & 0.1 & 1 & 1.1 & 0.006 & 1.299 & 0.421 & 0.579 & 0.7628 & 0.8144 & 0.7112 \\
0.8 & -0.1 & 0.1 & 1 & 1.1 & -0.314 & 1.422 & 0.199 & 0.801 & 0.6900 & 0.7709 & 0.6091 \\
0.9 & -0.1 & 0.1 & 1 & 1.1 & -2.040 & 1.143 & 0.174 & 0.826 & 0.6782 & 0.5641 & 0.7923 \\
\hline 0.9 & -0.2 & 0.2 & 1 & 1.2 & -1.739 & 1.071 & 0.202 & 0.798 & 0.5924 & 0.4814 & 0.7033 \\
\hline
\end{tabular}

We observe from Table 7 that for some percentiles the minimal efficiencies $u_{1}^{*}$ and $u_{2}^{*}$ in both models and thus their weighted means $u^{*}$ are substantially smaller compared to the results in Tables 1 and 3 which assume a fixed link function, whereas for other percentiles the loss in efficiency is much less severe. For example for $p=0.5$, i.e. estimation of the median, the optimal designs in the sense of (4.1) are still highly efficient, whereas optimal designs for the estimation of the $80 \%$-percentile exhibit significantly lower efficiencies $u_{1}^{*}, u_{2}^{*}$ and $u^{*}$. These results correspond to intuition, because the logistic and the normal distribution function with the same parameters are "close together" in the area around the mean but differ significantly elsewhere (see Figure 1). The maximin optimal designs for estimating the 50\%-percentiles in both models separately are thus also closer together than the corresponding designs for the 80\%-percentiles (see Tables 1 and 3) yielding a higher efficiency for the compromise design with respect to the criterion (4.1), which is in some sense situated between the two individual designs.

Using (4.1) as an optimality criterion, we thus gain model robustness of the design at the expense of efficiency with respect to the "true" model. There is, however, no robust alternative to this approach, particularly when keeping in mind that in most applications data are not "purely" either normal or logistic but something in between or even from a completely different (unknown) distribution family. 
Figure 1: The distribution functions of the logit and the probit model with mean zero and variance 1. Solid line: probit distribution. Dashed line: logit distribution.

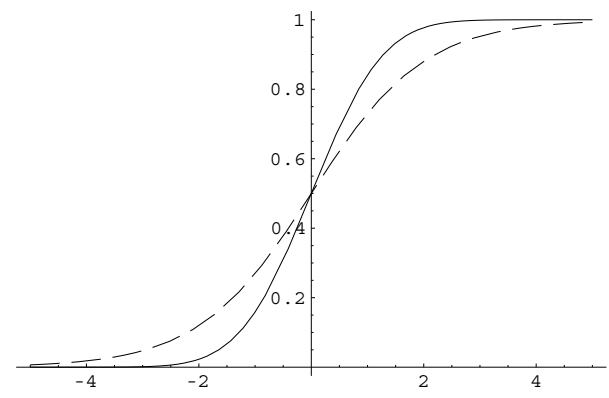

\section{Maximin efficient designs for estimating several per- centiles at the same time}

In this section, we consider a different aspect in the construction of robust designs, which is motivated by the observation that the experimenter may have several quantiles of interest. The goal here is to design the experiment efficiently for estimating several percentiles $x_{p}, p \in P$, at the same time, where $P$ is a subset of the interval $(0,1)$. We thus choose a prior distribution $\zeta$ on $P$ according to our preferences with respect to $p$, e.g., if we want to estimate the percentiles $x_{p_{1}}$ and $x_{p_{2}}$ "equally well", the uniform prior on $p_{1}$ and $p_{2}$ will be appropriate. In general, the optimality criterion is then given by the function

$$
\int_{P} \min _{\vartheta \in \Theta} \frac{c_{p}^{T} M^{-}\left(\xi_{c_{p}}^{*}, \vartheta\right) c_{p}}{c_{p}^{T} M^{-}(\xi, \vartheta) c_{p}} d \zeta(p) \rightarrow \max _{\xi}
$$

Obviously, this maximization problem is extremely hard to tackle in case of a continuous prior distribution $\zeta$. For calculations in practice, we therefore recommend to approximate a continuous prior $\zeta$ by a discrete distribution on $P$. For a discrete prior, however, we are in the same situation as in section 4 with the "prior" over the different possible link functions replaced by a prior on the percentiles to be estimated. The corresponding equivalence theorem, which is given below, is thus a generalization of Theorem 4.1 to the case of continuous prior distributions.

Theorem 5.1 Let $\zeta$ be a prior distribution on $P \subset(0,1)$. A design $\xi^{*}$ is optimal with respect to the criterion (5.1) if and only if for each $p \in P$ there exists a prior distribution $\pi_{p}^{*}$ on the set $\mathcal{N}_{p}\left(\xi^{*}\right)$ and for each $\vartheta \in \mathcal{N}_{p}\left(\xi^{*}\right)$ a generalized inverse $G\left(\xi^{*}, \vartheta\right)$ of the matrix $M\left(\xi^{*}, \vartheta\right)$ such that the inequality

$$
\int_{P} \int_{N_{p}\left(\xi^{*}\right)} \frac{c_{p}^{T} G\left(\xi^{*}, \vartheta\right) I(x, \vartheta) G\left(\xi^{*}, \vartheta\right) c_{p}}{c_{p}^{T} M^{-}\left(\xi^{*}, \vartheta\right) c_{p}} \operatorname{eff}_{c_{p}}\left(\xi^{*}, \vartheta\right) d \pi_{p}^{*}(\vartheta) d \zeta(p) \leq u^{*}
$$

holds for all $x \in \mathbb{R}$, where $\mathcal{N}_{p}\left(\xi^{*}\right)$ and $u^{*}$ are defined by

$$
\mathcal{N}_{p}\left(\xi^{*}\right)=\left\{\tilde{\vartheta} \in \Theta \mid \operatorname{eff}_{c_{p}}\left(\xi^{*}, \tilde{\vartheta}\right)=u_{p}^{*}\right\}
$$




$$
u^{*}=\int_{P} u_{p}^{*} d \zeta(p), \quad u_{p}^{*}=\min _{\vartheta \in \Theta} \operatorname{eff}_{c_{p}}\left(\xi^{*}, \vartheta\right) .
$$

Again, there is equality if $x$ is a support point of the design $\xi^{*}$.

In our numerical study, we consider situations, in which either two (see Tables 8 and 9) or three (see Table 10) different percentiles are of equal interest to the experimenter. Table 8 shows some representative results for the logit model, whereas Tables 9 and 10 give optimal designs with respect to the criterion (5.1) for the probit model. For the sake of brevity, only situations are displayed, where the optimal designs are supported at two points.

Table 8: Maximin efficient designs for the estimation of several percentiles at the same time in the logit model, $\zeta=\left\{p_{1}, p_{2} ; 1 / 2,1 / 2\right\}$.

\begin{tabular}{|c|c|c|c|c|c|c|c|c|c|c|c|c|}
\hline$p_{1}$ & $p_{2}$ & $\mu_{1}$ & $\mu_{2}$ & $\beta_{1}$ & $\beta_{2}$ & $x_{1}$ & $x_{2}$ & $w_{1}$ & $w_{2}$ & $u^{*}$ & $u_{p_{1}}^{*}$ & $u_{p_{2}}^{*}$ \\
\hline 0.5 & 0.6 & -0.1 & 0.1 & 1 & 1.1 & -0.504 & 0.972 & 0.496 & 0.504 & 0.7699 & 0.7608 & 0.7790 \\
0.6 & 0.7 & -0.1 & 0.1 & 1 & 1.1 & -0.535 & 1.106 & 0.314 & 0.686 & 0.7570 & 0.7460 & 0.7680 \\
0.7 & 0.8 & -0.1 & 0.1 & 1 & 1.1 & -0.779 & 1.341 & 0.188 & 0.812 & 0.7481 & 0.7341 & 0.7620 \\
0.8 & 0.9 & -0.1 & 0.1 & 1 & 1.1 & -1.364 & 1.667 & 0.142 & 0.858 & 0.7642 & 0.7291 & 0.7993 \\
\hline 0.5 & 0.7 & -0.1 & 0.1 & 1 & 1.1 & -0.704 & 1.213 & 0.417 & 0.583 & 0.6659 & 0.6569 & 0.6750 \\
0.5 & 0.8 & -0.1 & 0.1 & 1 & 1.1 & -0.881 & 1.409 & 0.394 & 0.606 & 0.5955 & 0.5984 & 0.5926 \\
0.5 & 0.9 & -0.1 & 0.1 & 1 & 1.1 & -1.110 & 1.608 & 0.371 & 0.629 & 0.5746 & 0.5258 & 0.6235 \\
\hline
\end{tabular}

As expected, we observe from Tables 8 and 9 a decline in minimal efficiency compared to the results in Tables 1 and 3, respectively, if the experiment is designed for estimating two different percentiles $x_{p_{1}}$ and $x_{p_{2}}$, where the decrease is even more significant if the distance between $p_{1}$ and $p_{2}$ is large. The last-mentioned effect obviously emerges from the fact that the optimal designs with respect to one percentile at a time are close if the distance between $p_{1}$ and $p_{2}$ is small and hence the optimal design for estimating both percentiles at the same time, which is in some sense situated between the other two designs, is not too far away from both of them. In general, all of the efficiencies displayed in Tables 8 and 9 are still significantly larger than $50 \%$ and thus in acceptable limits.

Table 10, finally, displays a somewhat further decline in minimal efficiency if the design is constructed for the efficienct estimation of three different percentiles simultaneously. We observe that the minimal efficiencies $u_{p_{1}}^{*}, u_{p_{2}}^{*}, u_{p_{3}}^{*}$ of these designs with respect to the estimation of one particular percentile are highest for the percentile corresponding to the value of $p_{2}$, which lies between the values $p_{1}$ and $p_{3}$. This effect probably occurs since designs, which are efficient for the simultaneous estimation of three percentiles with respect to values $p_{1}, p_{2}$ and $p_{3}$, will be compromise designs between the designs for the estimation of one of these percentiles at a time. The design that is maximin optimal for estimating the $100 p_{2}$ th percentile is in some sense located between the other two designs and the compromise design will therefore be close. 
Table 9: Maximin efficient designs for the estimation of several percentiles at the same time in the probit model, $\zeta=\left\{p_{1}, p_{2} ; 1 / 2,1 / 2\right\}$.

\begin{tabular}{|c|c|c|c|c|c|c|c|c|c|c|c|c|}
\hline$p_{1}$ & $p_{2}$ & $\mu_{1}$ & $\mu_{2}$ & $\beta_{1}$ & $\beta_{2}$ & $x_{1}$ & $x_{2}$ & $w_{1}$ & $w_{2}$ & $u^{*}$ & $u_{p_{1}}^{*}$ & $u_{p_{2}}^{*}$ \\
\hline 0.5 & 0.6 & -0.1 & 0.1 & 1 & 1.1 & -0.439 & 0.704 & 0.487 & 0.514 & 0.7863 & 0.7760 & 0.7966 \\
0.6 & 0.7 & -0.1 & 0.1 & 1 & 1.1 & -0.474 & 0.773 & 0.326 & 0.674 & 0.7724 & 0.7599 & 0.7849 \\
0.7 & 0.8 & -0.1 & 0.1 & 1 & 1.1 & -0.607 & 0.907 & 0.210 & 0.790 & 0.7562 & 0.7423 & 0.7702 \\
0.8 & 0.9 & -0.1 & 0.1 & 1 & 1.1 & -0.867 & 1.101 & 0.151 & 0.849 & 0.7462 & 0.7249 & 0.7675 \\
\hline 0.5 & 0.7 & -0.1 & 0.1 & 1 & 1.1 & -0.575 & 0.855 & 0.417 & 0.583 & 0.6967 & 0.6853 & 0.7081 \\
0.5 & 0.8 & -0.1 & 0.1 & 1 & 1.1 & -0.692 & 0.985 & 0.389 & 0.611 & 0.6254 & 0.6260 & 0.6248 \\
0.5 & 0.9 & -0.1 & 0.1 & 1 & 1.1 & -0.800 & 1.091 & 0.388 & 0.612 & 0.5840 & 0.5895 & 0.5785 \\
\hline
\end{tabular}

Table 10: Maximin efficient designs for the estimation of several percentiles at the same time in the probit model, $\zeta=\left\{p_{1}, p_{2}, p_{3} ; 1 / 3,1 / 3,1 / 3\right\},\left[\mu_{1}, \mu_{2}\right]=[-0.1,0.1],\left[\beta_{1}, \beta_{2}\right]=[1,1.1]$.

\begin{tabular}{|c|c|c|c|c|c|c|c|c|c|c|}
\hline$p_{1}$ & $p_{2}$ & $p_{3}$ & $x_{1}$ & $x_{2}$ & $w_{1}$ & $w_{2}$ & $u^{*}$ & $u_{p_{1}}^{*}$ & $u_{p_{2}}^{*}$ & $u_{p_{3}}^{*}$ \\
\hline 0.5 & 0.6 & 0.7 & -0.522 & 0.734 & 0.412 & 0.589 & 0.7355 & 0.7092 & 0.8317 & 0.6655 \\
0.6 & 0.7 & 0.8 & -0.580 & 0.822 & 0.293 & 0.707 & 0.7223 & 0.7097 & 0.8224 & 0.6348 \\
0.7 & 0.8 & 0.9 & -0.770 & 0.965 & 0.203 & 0.797 & 0.7115 & 0.6923 & 0.8057 & 0.6365 \\
0.5 & 0.7 & 0.9 & -0.742 & 0.901 & 0.348 & 0.652 & 0.6278 & 0.6093 & 0.7526 & 0.5216 \\
\hline
\end{tabular}

We must, however, keep in mind that the values presented in Tables 8-10 are only minimal efficiencies, i.e. they give only the efficiencies for the worst case scenario with respect to $\Theta$. If other values from $\Theta$ are the "true" parameters in our model, the efficiency of the designs will be significantly higher. Consider, for example, the parameter combination $\mu=0, \beta=1.05$, i.e. the midpoint of the rectangle $\Theta$. Assume, furthermore, the percentiles corresponding to $p_{1}=0.8$ and $p_{2}=0.9$ are equally important and the logit link function describes the model appropriately. Then the value of the overall efficiency of the optimal design with respect to the criterion (5.1) is given by 0.829 , which is considerably higher compared to its minimal efficiency 0.764 from Table 8 . The efficiencies with respect to one percentile at a time are given by 0.797 and 0.860 , respectively, which are also higher than the corresponding minimal values 0.729 and 0.799 .

\section{Conclusions}

In this article, we discuss several aspects of robust design of experiment for estimating the effective dose level in binary response models. In general, optimal designs minimize an approximation of the variance of the maximum likelihood estimate for the $100 p$ th percentile $x_{p}$ and depend sensitively on the unknown parameter, the link function and the quantile of interest. 
Several strategies based on the concept of maximin efficiency are discussed to obtain designs which are on the one hand efficient for the effective dose level and on the other hand robust with respect to these assumptions. It is demonstrated that robust designs with respect to the new criteria can be calculated numerically and that the optimality of these designs can be checked by non-standard equivalence theorems. The most important robustification is obtained assuming that the experimenter is only able to specify a certain range $\Theta$ for the unknown parameters and it is demonstrated that the maximin efficient designs are efficient for all values in $\Theta$, provided that this set is not too large. These designs are then further robustified with respect to the assumptions on the link function (section 4) and the particular quantiles $x_{p}$ chosen by the experimenter.

It is also -theoretically and numerically- possible to combine the approaches of sections 4 and 5 to achieve a further robustification of the designs, i.e. to define an optimality criterion, which is robust with respect to misspecifications of the parameters, the binary response model and at the same time efficient for the estimation of several percentiles. This criterion corresponds in some sense to a combination of maximin- and Bayesian criteria over the parameter space $\tilde{\Theta}=(\Theta \times \mathcal{F} \times \mathcal{P})$, where $\mathcal{F}$ denotes the class of reasonable link functions and $P \subset(0,1)$ a set of percentiles of interest. One should, however, keep in mind that adding extra dimensions to the possible alternatives, against which the design of the experiment has to be robustified, has the same effect on the efficiency of the resulting designs as enlarging its individual components, i.e. the (minimal) efficiencies will decrease as can already be seen from the examples in Tables 7-10. Robustification of designs will always be bought at the expense of a loss in efficiency. The experimenter should therefore carefully deliberate about the question which amount of robustness is necessary for an appropriate data analysis.

The discussion at the end of section 3, however, shows that there is no robust alternative to the maximin approach with respect to the parameter region $\Theta$. In particular, it turns out that the less information about the approximate position of the unknown parameter $\vartheta$ is available (i.e. the larger the region $\Theta$ is chosen), the less robust against particular values of $\vartheta \in \Theta$ the Bayesian $c_{p}$-efficient design (with respect to the uniform prior on $\Theta$ ) will be. Consequently, the more uncertainty about the parameter $\vartheta$ exists, the stronger the maximin approach is recommended.

Acknowledgements: The support of the Deutsche Forschungsgemeinschaft (SFB 475, Komplexitätsreduktion in multivariaten Datenstrukturen, Teilprojekt A2, Sachbeihilfe 436 RUS 113/712/1-1) is gratefully acknowledged.

\section{References}

[1] Chaloner, K. and Larntz, K. (1989). Optimal Bayesian experimental design applied to logistic regression. Journal of Statistical Planning and Inference, 21, 191-208.

[2] Chernoff, H. (1953). Locally optimal designs for estimating parameters. Annals of Mathematical Statistics, 24, 586-602. 
[3] Dette, H. and Biedermann, S. (2003). Robust and efficient designs for the MichaelisMenten model. Journal of the American Statistical Association, 98, 679-686.

[4] Dette, H. and Wong, W.K. (1996). Optimal Bayesian designs for models with partially specified heteroscedastic structure. Annals of Statistics, 24, No. 5, 2108-2127.

[5] Dette, H., Haines, L. and Imhof, L. (2003). Maximin and Bayesian optimal designs for regression models. preprint. http://www.ruhr-uni-bochum.de/mathematik3/preprint.htm

[6] Dette, H. (1997). Designing experiments with respect to standardized optimality criteria. Journal of the Royal Statistical Society, Ser. B, 59, No.1, 97-110.

[7] Fandom Noubiap, R. and Seidel, W. (2000). A minimax algorithm for constructing optimal symmetrical balanced designs for a logistic regression model. Journal of Statistical Planning and Inference, 91, No. 1, 151-168.

[8] Ford, I., Torsney, B. and Wu, C.F.J. (1992). The use of a canonical form in the construction of locally optimal designs for non-linear problems. Journal of the Royal Statistical Society, 54, 569-583.

[9] Läuter, E. (1974). Experimental design in a class of models. Mathematische Operationsforschung und Statistik, 5, 379-398.

[10] López-Fidalgo, J. and Wong, W.K. (2000). A comparative study of MV-and SMV-optimal designs for binary response models. In: Advances in stochastic simulation methods (ed. N. Balakrishnan), 135-151, Birkhäuser, Boston, USA.

[11] Müller, C. H. (1995). Maximin efficient designs for estimating nonlinear aspects in linear models. Journal of Statistical Planning and Inference, 44, 117-132.

[12] Pukelsheim, F. (1993). Optimal Design of Experiments. Wiley, New York.

[13] Sitter, R.R. (1992). Robust Designs for Binary Data. Biometrics, 48, 1145-1155.

[14] Sitter, R.R. and Wu, C.F.J. (1993). Optimal designs for binary response experiments: Fieller-, D- and A-criteria. Scandinavian Journal of Statistics, 20, 329-341.

[15] Wu, C.F.J. (1985). Efficient sequential designs with binary data. Journal of the American Statistical Association, 80, 974-984.

[16] Wu, C.F.J. (1988). Optimal design for percentile estimation of a quantal response curve. Optimal design and analysis of experiments. Amsterdam: Elsevier (North Holland).

[17] Zhu, W. and Wong, W.K. (2000). Multiple-objective designs in a dose-response experiment. Journal of Biopharmaceutical Statistics, 10, No. 1, 1-14. 